Thorax $1981 ; 36: 721-725$

\title{
Editorial
}

\section{Mast cells and basophils: effector cells of inflammatory disorders in the lung}

The past several years have seen a number of new and exciting discoveries which have increased our knowledge of the mediators that may be operative in immune disorders of the lung, such as bronchial asthma. In this editorial, we would like to place these new observations in perspective and, particularly, to point out some of the complexities arising from these data and to emphasise the questions which remain to be resolved.

Perhaps the most exciting new data relate to the chemical delineation of several mediators. This has been an arduous task. In the case of the slowreacting substances of anaphylaxis (SRS-A), research was begun even before 1940 when Kellaway and Trethewie showed that this lipid mediator was involved in anaphylactic reactions. ${ }^{1}$ Many investigators provided data that led in 1979 to the publication by Samuelsson and his colleagues which indicated that the leukotrienes (SRS-A) are products of arachidonic acid via the lipoxygenase pathway. ${ }^{2}$ A more recently discovered mediator, platelet activating factor, was first described in $1971 .^{3}$ In the last year, the structure of this mediator has also been elucidated by Pinckard and Hanahan: it is another lipid, acetyl glyceryl ether phosphorylcholine (AGEPC). ${ }^{4}$

In attempting to discern the in vivo importance of such mediators, it would appear that we have an embarrassment of riches: the number of mediators which can cause bronchospasm, or other events which appear related to IgE mechanisms, suggest that the system is redundant. Thus, histamine in many species and in man can reproduce most or all of the symptoms of immediate hypersensitivity reactions. Studies by Pinckard and colleagues, in rabbits and baboons, suggest that AGEPC can also produce bronchospasm and hypotension. The leukotrienes also have a wide variety of actions and may account for a considerable part of the respiratory distress which characterises the response of man to antigens. One must not, however, forget the older studies which show that antigen-challenged, sensitised guinea pigs die of acute bronchospasm and that this response is completely ablated by antihistamines.

Address for reprint requests: Dr Harold $\mathrm{H}$ Newball, The Johns Hopkins University School of Medicine, O'Neill Research Laboratories, 5601 Loch Raven Boulevard, Baltimore, Maryland 21239, USA.

\section{Modulation of mediator release}

Sensitised mast cells and basophils, when challenged with the appropriate antigens, release several low molecular weight mediators (histamine, the leukotrienes, prostaglandins, chemotactic factors, and so on), and high molecular weight mediators. ${ }^{5-7}$ The processes which are involved in mediator release have been extensively described. In each instance, the mediator is actively secreted by a process that is temperature and calcium-dependent, requires metabolic energy, and is modulated by hormone-receptor interactions that influence the intracellular level of cyclic nucleotides. ${ }^{89}$ Agonists which act on specific receptors to activate adenylate cyclase and increase intracellular levels of cyclic AMP inhibit the release of mediators. Thus, prostaglandin E, beta-adrenergic agonists, histamine (in the case of basophils but not lung mast cells), and adenosine inhibit the release of mediators. Agents such as theophylline and IBMX (isobutylmethylxanthine) which exert their pharmacological effects through the inhibition of phosphodiesterase with a consequent increase of intracellular cyclic AMP, likewise inhibit the release of mediators. Colchicine, which causes disaggregation of microtubules, is a potent inhibitor of mediator release while heavy water $\left(\mathrm{D}_{2} \mathrm{O}\right)$ which favours microtubular aggregation is a potent enhancer of mediator release. Mediator release is an active process. It has been demonstrated that, in the secretory process, there is an absolute requirement for metabolic energy and, if glycolytic processes are impaired, mediator release ceases. Thus, metabolic inhibitors such as 2-deoxyglucose will inhibit the release of mediators from mast cells and basophils. ${ }^{8}$

One of the most interesting recent observations with respect to the release of several chemical mediators from mast cells and basophils has to do with the role of arachidonic acid metabolism in the release process. With respect to histamine release, it has been shown that eicosatetraenoic acid (ETYA), which blocks both the lipoxygenase and the cyclooxygenase pathways of arachidonic acid metabolism completely inhibits the release of histamine. On the other hand, the non-steroidal anti-inflammatory drugs such as indomethacin, aspirin, and meclofenamic acid, which are selective inhibitors of the cyclooxygenase pathway of arachidonic metabolism, potentiate the release of histamine. These observa- 
tions suggest that a product of the lipoxygenase pathway is necessary for histamine release, and that the nonsteroidal anti-inflammatory drugs act by shunting arachidonic acid metabolism to the lipoxygenase pathway. In keeping with this suggestion is the fact that arachidonic acid itself also potentiates histamine release. A related observation with respect to the above data is that indomethacin and other nonsteroidal anti-inflammatory drugs selectively block the inhibition of histamine release caused by each of the agonists (isoproterenol, histamine, adenosine, prostaglandins) which act through specific receptors to increase adenylate cyclase activity. Arachidonic acid has a similar activity. On the other hand, neither indomethacin nor arachidonic acid has any effects on the inhibition of histamine release caused by drugs or agents which increase cyclic AMP by other mechanisms. Thus, indomethacin and arachidonic acid do not reverse the inhibition of histamine release caused by theophylline or isobutylmethylxanthine; similarly, they have little or no effect on the inhibition of histamine release caused by dibutyryl cyclic AMP itself. These data strongly suggest that there must be subcompartments of cyclic AMP which are differently affected by cyclase agonists and by other agents which increase cyclic AMP. They further suggest that, not only is there a product of the lipoxygenase pathway that is necessary for histamine release, but that another product of that pathway modulates the effects of endogenous hormones or pharmacological agents on the control of histamine release. We have recently investigated the influence of several lipoxygenase products (HPETE, 5-HETE, LTC 4, LTD $_{4}$, and so on) on the release of histamine from suspensions of basophils and human lung mast cells. The data suggest that 5-HPETE may function in an important aspect of the modulation of histamine release from basophils.

\section{Low molecular weight mediators}

Also related to arachidonic acid metabolism is the recently described structure of the leukotrienes (SRS-As). The IgE-mediated release of several forms of biologically active leukotrienes (SRS-As) from human lung was first reported many years ago. ${ }^{10}$ However, it was only recently shown that the leukotrienes are derived from the lipoxygenase pathway of arachidonic acid metabolism and that there are at least three biologically active compounds (leukotrienes C, D, and E). ${ }^{11} 12$ Another interesting observation is that the major source of the leukotrienes in inflammatory reactions may not be basophils or mast cells, but rather other cell types. Thus, Bach has shown that SRS may be derived from rat peritoneal mononuclear cells, ${ }^{13}$ and we have demonstrated that SRS is readily derived from human polymorphonuclear leucocytes. The precise $\overline{ }$ role of the leukotrienes in inflammatory disorders of $\frac{\bar{s}}{\square}$ the lung is not fully elucidated but, with respect to $\stackrel{\mathbb{Q}}{\propto}$ airway smooth muscle, the duration of its action is known to be much longer than that of histamine. The definition of the structure of the leukotrienes will, for the first time, allow the synthesis of a series $\overrightarrow{\vec{\omega}}$ of antagonists which may delineate the role of this mediator in inflammatory processes of the lung.

There are a number of eosinophil chemotactic $\dot{\omega}$ factors which have been reported to be derived from mast cells and basophils. Two of these have been characterised as tetrapeptides by Goetzl and $N$ Austen; ${ }^{14}$ however, here as well, there is the obser- $\vec{\circ}$ vation that eosinophil chemotactic factors may be $\frac{}{\triangle}$ readily obtained from polymorphonuclear leuco- $\vec{\circ}$ cytes when they are challenged with the calcium ionophore, or as a result of phagocytic stimuli. ${ }^{15} \frac{O}{O}$ There is also recent evidence that arachidonic acid $\stackrel{\mathbb{Q}}{-}$ is the precursor of an important chemotactic factor. $\vec{\varphi}$ The studies by Goetzl and coworkers have shown $\stackrel{\infty}{\rightarrow}$ that LTB $_{4}(5,12$-di-HETE) is derived from arachi- 0 donic acid via the lipoxygenase pathway. Microgram quantities of 12-HETE, and 5-HETE, and nanogram quantities of 5,12-di-HETE $\left(\mathrm{LTB}_{4}\right)$ are capable of eliciting a rapid accumulation of eosinophils, and a $\frac{\varnothing}{\varnothing}$ delayed accumulation of neutrophils. ${ }^{16}$ Thus, yet $\stackrel{\varrho}{\rightleftharpoons}$ another important metabolite of arachidonic acid $\overline{0}$ has been shown to have the potential of an important inflammatory mediator.

Platelet activating factor (AGEPC) is another low molecular weight mediator, whose structure has recently been elucidated. ${ }^{4}$ The effects of AGEPC on the human respiratory system are unknown. There ${ }_{-}^{x}$ have, however, been extensive studies on the physio- 3 . logical effects of AGEPC on rabbits and baboons. It has become evident that AGEPC is a very impor- $\frac{3}{3}$ tant mediator, with physiological effects being evident at the nanomolar level. In lower species, AGEPC is capable of increasing pulmonary resistance and decreasing pulmonary compliance. At a dose of $0.6 \mu \mathrm{g} / \mathrm{kg}$, AGEPC was observed to produce $\widetilde{N}$ physiological alterations in the lung which were both $N$ qualitatively and quantitatively indistinguishable from those which characterise IgE anaphylaxis in ${ }_{\sigma}^{\omega}$ the rabbit. ${ }^{17}$ If AGEPC participates in anaphylactic reactions in man, as it does in rabbits, the results of Pinckard and associates provide strong evidence that AGEPC has the potential to be an important ${ }_{7}^{-}$ mediator of allergic and inflammatory responses in the human lung.

\section{High molecular weight mediators}

It appears that the low molecular weight mediators that we have described (histamine, leukotrienes, 
$\mathrm{LTB}_{4}, \mathrm{AGEPC}$ ) are responsible for but a part of the symptom complex observed during reactions of the lung such as those observed during anaphylaxis and inflammatory disorders. Consider, for example, the least complex type of allergic reaction, anaphylaxis, which we have studied as part of our work on insect allergy. This reaction is solely caused by $\operatorname{IgE}$ antibody and to a first approximation, the degree of hypotensive shock seems to parallel the plasma histamine level. A closer examination of physiological responses, however, indicates that many other events are probably occurring during anaphylaxis. We have recently published data which indicate that, during human anaphylaxis, intravascular coagulation occurs, with depletion of coagulation factors. There is also consumption of high molecular weight kininogen, $\mathrm{C} 4$ and $\mathrm{C} 3$. The mediators which account for the activation of the coagulation system have not been defined. We have recently, however, reported the IgE-mediated release of three proteases from antigen-challenged passively sensitised human lung. ${ }^{18}$ One of these proteases (figure) activates Hageman factor, another activates prekallikrein, and a third cleaves high molecular weight kininogen to generate a biologically active kinin. The figure illustrates how these newly described protease mediators may account for at least some of the changes observed in the coagulation proteins, which appear to participate in the physiological events observed during anaphylaxis.

\section{Mechanisms of mediator release}

There have been extensive studies into the mechanism by which chemical mediators are released from mast cells and basophils. The crosslinking of IgE molecules on the surface of mast cells and basophils leads to the initiation of two distinct processes, the outcome of which determines the extent of mediator release. There is first an activation pathway which leads eventually to the exocytosis of mast cell and basophil granules. Simultaneously, however, a desensitisation process is set in motion which acts to stop the release of mediators. Recent work in our laboratories has shown that desensitisation of two different types can occur. When a small number $(<10000)$ of crosslinked triggering units results from an antigenic stimulus, specific desensitisation occurs. That is, the cells are rendered specifically unresponsive to a particular antigen, but are able to respond to other antigens or to anti-IgE. Specific desensitisation leads to inactivated triggering units, but the remaining units of other specificities remain fully operative. When, however, the number of crosslinked triggering units is higher ( $>30000)$, non-specific desensitisation occurs, such that the cell is now unresponsive to any IgE-mediated stimulus. ${ }^{19}$ The mechanism for this non-specific desensitisation is not clear, but a variety of studies suggests that it involves the closing of calcium gates, the hypothetical structure which allows the influx of calcium, upon which release of mediators is totally dependent. If the desensitisation process does not predominate, activation proceeds to histamine release through an extremely complex set of reactions which are only beginning to be appreciated. Control of the relative degree of activation versus desensitisation can have an important influence on the extent of the inflammatory response.

\section{Summary}

It is possible to summarise what is now known about the biochemical mechanism of mediator release in the following fashion, recognising that this simply represents the present state of the art. The cross-

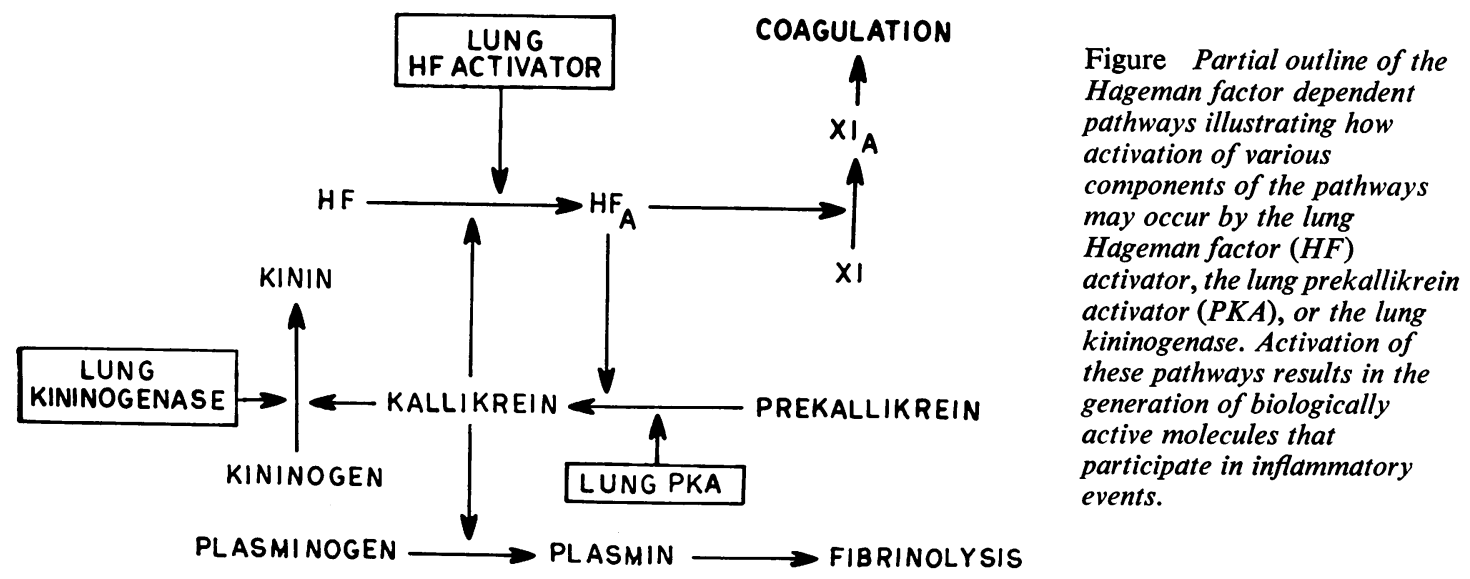

Figure Partial outline of the Hageman factor dependen pathways illustrating how activation of various may occur by the lung Hageman factor $(H F)$ activator, the lung prekallikrein activator $(P K A)$, or the lung kininogenase. Activation of participate in inflammatory events. 
linking of $\operatorname{IgE}$ receptors leads to a change in the intracellular activity of adenylate cyclase and an increase in membrane phospholipid transmethylation. ${ }^{20}$ Through as yet unknown mechanisms, this is associated with, or causes, an increase in the permeability of the cell to calcium, perhaps via a modulatory protein which serves as the interface between receptor bridging and the opening of the postulated calcium channels. It is clear, at least in human cell types, that these processes can be antagonised by hormone-receptor interactions which lead to the stimulation of adenylate cyclase and an increase in intracellular cyclic AMP. The above events occur early in the secretory process. Later on in the sequence of reactions are the changes in phospholipid biosynthesis and the release of arachidonic acid. It seems likely that the latter event follows the activation of phospholipase A, although other enz.ymes have been implicated. Steroids, which inhibit histamine release with a time course reminiscent of their in vivo anti-inflammatory activity, may well act through the induction of a protein which blocks phospholipase A activation. It appears clear that one of the products of arachidonic acid metabolism, via the lipoxygenase pathway, is necessary for histamine release. These data were obtained by the inhibition of histamine release by specific and nonspecific lipoxygenase inhibitors. Moreover, we have identified a product of the lipoxygenase pathway, 5-hydroperoxy-eicosatetraenoic acid, which has this activity. Thus, the effects of indomethacin on histamine release are caused by the shifting of the metabolism of arachidonic acid from the cyclooxygenase to the lipoxygenase pathway. All of these biochemical changes lead, in a yet undefined way, to changes in the cytoskeletal elements of the cell which cause an energy dependent exocytosis of basophil and mast cell granules and, thus, the release of mediators.

Both the identification of the mediators of allergic reactions, with the potential for the synthesis of antagonists, and the delineation of biochemical pathways, with the potential for developing pharmacological agents which block the entire release mechanism, suggest that the next decade of studies on the mechanism and therapy of bronchial asthma and other immune disorders of the lung, will be fruitful. It should be recognised, however, that immune disorders of the lung are extremely complex and that we have only just begun to understand the biochemical nature of mediators and the mechanisms of their release.

HHN was supported by grant number HL24210 from the NHLBI, NIH, and grant number 1392 from the Council for Tobacco Research, USA Inc.

HAROLD H NEWBALL LAWRENCE M LICHTENSTEIN

\author{
Department of Medicine, \\ The Johns Hopkins University School \\ of Medicine, Baltimore, USA
}

References

${ }^{1}$ Kellaway $\mathrm{CH}$, Trethewie ER. Liberation of slow reacting $\vec{O}$ smooth muscle stimulating substance in anaphylaxis. Q J Exp Physiol 1940;30:121-45.

${ }^{2}$ Murphy RC, Hammarstrom S, Samuelsson B. Leukotriene C. A slow-reacting substance from murine mastocytoma cells. Proc Natl Acad Sci USA 1979;76:4275-9.

${ }^{3}$ Siraganian RP, Osler AG. Destruction of rabbit platelets in the allergic response of sensitized leukocytes. I. Demonstration of a fluid phase intermediate. $J$ Immunol $1971 ; 106: 1244-51$.

${ }^{4}$ Demopoulos CA, Pinckard RN, Hanahan DJ. Plateletactivating factor: Evidence for 1-0-alkyl-2-acetyl-sn- 윽 glyceryl-3-phosphorylcholine as the active component (a new class of lipid chemical mediators). J Biol Chem 1979;254:9355-8.

${ }^{5}$ Newball HH, Meier HL, Lichtenstein LM. Basophil mediators and their release, with emphasis on BK-A. $J$ Invest Derm 1980;74:344-8.

- Newball HH, Berninger RW, Talamo RC, Lichtenstein LM. Anaphylactic release of a basophil kallikrein-like activity. I. Purification and characterization. J Clin Invest $1979 ; 64: 457-65$.

? Adkinson NF, Newball HH, Findlay S, Adams GK Lichtenstein LM. Anaphylactic release of prostaglandins from human lung in vitro. Am Rev Respir Dis 1980;121: 911-20.

${ }^{8}$ Newball HH, Talamo RC, Lichtenstein LM. Anaphylactic release of a basophil kallikrein-like activity. II. A mediator of immediate hypersensitivity reactions. J Clin Invest $1979 ; 64: 466-75$.

${ }^{9}$ Lichtenstein LM. Mechanisms of allergic inflammation. In: Mandel TE et al, eds. Progress in Immunology III. Amsterdam: North Holland Publishing, 1977:430-8.

10 Takahasi H, Webster ME, Newball HH. Purification of slow reacting substance of anaphylaxis (SRS-A) from human lung. $J$ Immunol 1976;117:1039-44.

${ }^{11}$ Parker CW, Huber MM, Hoffman MK, Falkenheim SF. Characterization of the two major species of slow reacting substance from rat basophilic leukemia cells as glutathionyl thioethers of eicosatetraenoic acids oxygenated at the 5 position: evidence that peroxy groups are present and important for spasmogenic activity. Prostaglandins 1979;18:673-86.

12 Orning L, Hammarstrom S, Samuelsson B. Leukotriene D: a slow reacting substance from rat basophilic leukemia cells. Proc Natl Acad Sci USA 1980;77:2014-7.

${ }^{13}$ Bach MK, Brashler JR. Ionophore A 23184-induced production of slow reacting substance of anaphylaxis (SRS-A) by rat peritoneal cells in vitro: evidence for production by mononuclear cells. J Immunol $1978 ; 120$ : 998-1005.

14 Goetzl EJ, Austen FK. Purification and synthesis of eosinophilotactic tetrapeptides of human lung tissue: identification as eosinophil chemotactic factor of anaphylaxis. Proc Natl Acad Sci USA 1975;72:4123-7.

15 Konig W, Czarnetzki BM, Lichtenstein LM. Eosinophil chemotactic factor (ECF). II. Release from human polymorphonuclear leukocytes during phagocytosis. $J$ Immunol 1976;117:235-41.

16 Goetzl EJ. Mediators of immediate hypersensitivity derived from arachidonic acid. $N$ Engl J Med 1980;303: 822-5. 
${ }^{17}$ Halonen M, Palmer JD, Lohman IC, McManus LM, Pinckard RN. Respiratory and circulatory alterations induced by acetyl glyceryl ether phosphorylcholine, a mediator of IgE anaphylaxis in the rabbit. Am Rev Respir Dis 1980;122:915-24.

18 Newball HH, Meier HL, Kaplan AP, Revak SD, Cochrane CG, Lichtenstein LM. Activation of the Hageman factor dependent systems during IgE-mediated reactions of the human lung. Int Arch Allergy Appl Immunol 1981 ; in press. ${ }^{19}$ Sobotka AK, Dembo M, Goldstein B, Lichtenstein LM. Antigen-specific desensitization of human basophils. $J$ Immunol 1979;122:511-7.

${ }^{20}$ Ishizaka T, Hirata F, Ishizaka K, Axelrod J. Stimulation of phospholipid methylation, $\mathrm{Ca}^{2+}$ influx, and histamine release by bridging of IgE receptors on rat mast cells. Proc Natl Acad Sci USA 1980;77:1903-6. 